\title{
Clinical and Pathohistological Profiles of Chronic Odontogenic Infections with Microvascular Proliferation in Children with Permanent Dentition
}

\author{
Teuta Ademaj Kutllovci' ${ }^{1}$, Snezana Iljovska ${ }^{2}$, Agim Begzati ${ }^{1}$, Aida Rexhepi ${ }^{1}$, \\ Blerta Latifi-Xhemajli ${ }^{1}$, Urata Tahiri ${ }^{1}$ \\ ${ }^{1}$ Department of Pediatric Dentistry, University Dental Clinic of Kosova, Pristina, Kosovo \\ ${ }^{2}$ Department of Pediatric Dentistry, Ss. Cyril and Methodius University in Skopje, Skopje, Macedonia \\ Email: teutakutllovci62@hotmail.com, iljovskasnezana@yahoo.com, agimbegzati@yahoo.com, \\ aida.rexhepi@hotmail.com, blertalatifipr@gmail.com, teutakutllovci62@hotmail.com
}

Received 26 February 2015; accepted 7 April 2015; published 10 April 2015

Copyright (C) 2015 by authors and Scientific Research Publishing Inc. This work is licensed under the Creative Commons Attribution International License (CC BY). http://creativecommons.org/licenses/by/4.0/

\section{(c) (i) Open Access}

\section{Abstract}

The apical periodontitis as an infection is the result of endodontic infections and is manifested when the immunological system of the host is activated from microorganisms which penetrate in to the dental pulp through the root canals. The aim of this study is to compare the clinical diagnosis and the pathohistological identification of chronic odontogenic infections with microvascular proliferation. The study was carried out in University Dental Clinical Center of Kosova in cooperation with Histopathology Departement. In this study were included 36 children between 10 - 15 years old, 20 girls and 16 boys, with permanent dentition and chronic apical changes. After the clinical and $x$-ray examination, the tooth extraction was done, and then the tissue of periapical region was taken, by the exvaction of the periapical granulomas and the other pathological tissues. From a total of 36 children with chronic apical changes and granulomas, $9(25.0 \%)$ of the children had chronic apical periodontitis, from which $6(16.67 \%)$ of the children did not have microvascular proliferation, $1(2.78 \%)$ child had a medium level of microvascular proliferation and $2(5.56 \%)$ children had signification microvascular changes (proliferation). A total of $6(16.67 \%)$ children had chronic apical periodontitis with a fistula from which 5 (13.89\%) children did not have microvascular proliferation and $1(2.78 \%)$ child had a medium level of microvascular proliferation. A total of $5(13.89 \%)$ children had chronic apical periodontitis with parulis, from which 3 (8.33\%) children did not have microvascular proliferation, 1 (2.78\%) child had a low level of microvascular proliferation and $1(2.78 \%)$ child had severe microvascular proliferation. 


\section{Keywords}

\section{Chronic Odontogenic Infections, Pathohistological Examinations, Apical Periodontitis, Microvascular Proliferation}

\section{Introduction}

The apical periodontitis as an infection is the result of endodontic infections and is manifested when the immunological system of the host is activated from microorganisms which penetrate in to the dental pulp through the root canals.

Dental caries is a disease which nowadays is considered to have a high prevalence like the cardiovascular and psychiatric diseases [1]. The odontogenic infections are not a singular specific pathology and they are always a result of complications of untreated dental caries as a common factor or they are a result of other predisposing factors [1]-[5].

The grown and evolution of the orofacial systems in whole, the evolution and maturation processes of the dento-alveolar tissues and the evolutional processes of the primary and the young secondary dentition have a great role in the initiation and evolution of the odontogenic infection in children. The diseases of the dental pulp and the periodontal tissues are a result of the anatomical, physiological and histological characteristics [6]. The causes which have a role in the initiation of the pathological processes in the pulp can be :microbiological (bacteria and their toxins), chemical (medications, the materials used in dental fillings and the alcohol used to clean the dental cavity), mechanical (they can be: acute—insensitive work with instruments and chronic —bad habits, bruxism, inadequate orthodontic braces, hot or cold drinks, galvanic microcurrent, improper and frequent usage of vitalion) and iatrogenic, from which the most common are rough usage of the rotating instruments, prolonged drying of the cavities, artificial opening of the pulp and exc [7] [8].

Stashenko said that the infections of the bone structures and the soft tissues of the oral cavity most commonly have dental etiology but at the same time other factors such as the haematogenous, lymphatic and traumatic components can have an influence [9].

The apical periodontitis is a result of an endodontic infection and it is manifested in those moments when the immunological system of the host is activated from the microorganisms which invade from the root canals [10]. The evolution of the apical periodontitis is considered as a dynamic confrontation between the microbiological factors and the immunological mechanism of the host in the region between the infected radicular pulp and the periodontal ligament which results with a local inflammation, resorpsion of the hard tissue, destruction of the other periodental tissues and eventually forming of different histopathological categories of the apical periodontitis, frequently named as periapical lesions [10].

Very little attention is given to the need of pathohistological examination of the periapical tissues outside of hospitals. Many authors ( 9 - 13) have said that the periapical lesions which do not react to the conservative endodontic treatment should undergo pathohistological verification. The guidelines from the American Association of Endodontists suggest that a pathohistological verification should always be done whenever we have enough tissue which can be processed. One author who believes that the clinical diagnosis has a high percent of accuracy states that a careful systematic clinical diagnosis can differentiate the endodontic from the non-endodontic pathological processes and that the routine sending of surgical material for pathohistological diagnosis does not have any advantage. The decision for taking material for a biopsy is made in the moment when we have a clinical suspicion and we want to evade setting a wrong diagnosis, which can be evaded by sending the tissue for pathohistological verification [11].

Taking into consideration the stages of the inflammation of the dental pulp and surrounding structures and the everyday problems which we encounter in our dental offices and the dilemmas which come when we are deciding about a correct diagnosis, we set goals with which we can compare the clinical diagnosis and the pathohistological identification in chronic dental infections with microvascular proliferation in kids with permanent dentition.

\section{Purpose of the Study}

Due to the high number of the patients with chronic odontogenic infections and periapical changes and granu- 
lomas identified by retroalveolar $\mathrm{x}$-ray, the purpose of our study was to compare the clinical diagnosis of chronic odontogenic infections and the pathohistological identification of this infections with microvascular proliferation in different group of children.

\section{Methods and Materials}

For the realization of our goals we included 36 children, age 10 - 15 years old, 20 girls and 16 boys, all of them with chronic apical processes and granulomas were indicated for extraction of the tooth because of the dental infections. All children were divided to 4 groups, depending from clinical diagnosis for which have been notified, examined and according to microvascular proliferation level found on histopathological preparations. The research has been finished in University Dental Clinical Center of Kosova, Prishtine, Kosove, period of JanuarySeptember 2014.

Before tooth extraction, a written consent was taken from the parents of the children who was noted in a specialized questioner. The research has been approved from Ethical Proffesional Committee of UDCCK (University Dental Clinical Center of Kosova).

The number of children participating in this research is compatible with the results of the study.

\subsection{Pathohistological Examinations}

In the tooth in which there was a chronic processes and we suspected a granuloma, in which tooth we determined the existence of a granuloma with an $x$-ray, after the extraction of the tooth we took a piece of the tissue with a sterile scalpel and we placed it in formalin. These samples were sent to the Institute for Pathology where after 24 hours the samples were made into samples with a thickness of $4 \mathrm{~mm}$. These samples were placed in special cassettes in which they stayed for another 24 hours, after which they were put in a Histokinet machine for another 24 hours. After this time the slides were put in paraffin so they can be prepared into samples with a thickness of 4 microns. These samples were then put into special dies in which they stayed for 30 minutes. After they dried they were covered with special covering glasses and the results were read by a pathologist.

The pathohistological evaluation was done at the Institute for Pathology in the Medical Faculty in Prishtina.

\subsection{Statistical Analysis}

The analyses of the data were done in the statistical program Statistica 7.1. The association between the clinical diagnosis and the pathohistological diagnosis was tested with the x2 test/Fisher. A risk of inflammation, edema, hyperemia, microvascular proliferation, hemorrhage, and fibrosis was assessed with OR/Wald 95\%Cl. The significance was determined for $\mathrm{p}<0.05$.

\section{Results}

In Table 1, we can see the distribution of the data in correlation with the clinical diagnosis and the pathohistological diagnosis in the group of children with chronic apical processes and granulomas. From a total of 36 children with chronic apical processes and granulomas, 9 (25\%) of the children had chronic apical periodontitis, from which 6 (16.67\%) of the children did not have microvascular proliferation and 2 (5.56\%) of children had expressed microvascular proliferation. Total 16 (44.44\%) children had chronic apical periodontitis with granulomas from which 1 (2.78\%) child did not have microvascular proliferation, 4 (11.11\%) children had a low level microvascular proliferation, 6 (16.67\%) children had a middle level microvascular proliferation and 5 (13.89\%) children had a high level microvascular proliferation.

A total of 6 (16.67\%) children had chronic apical periodontitis with a fistula from which 5 (13.89\%) children did not have microvascular proliferation and $1(2.78 \%)$ child had a middle level microvascular proliferation. A total of 5 (13.89\%) children had chronic apical periodontitis with parulis, from which 3 (8.33\%) children did not have microvascular proliferation, 1 (2.78\%) child had a low level microvascular proliferation and $1(2.78 \%)$ child had a high level microvascular proliferation. The results showed that $\mathrm{p}>0.05$ Fisher exact/and that there is a correlation between clinical diagnosis and pathohistological diagnosis respectively and microvascular proliferation.

The data in Table 2 show the association between the chronic apical periodontitis and microvascular proliferation. From a total of 36 children, chronic apical periodontitis had 9 (25.00\%) children, from which 3 (8.33\%) children had microvascular proliferation and 6 (16.67\%) children did not have microvascular proliferation. A 
Table 1. Clinical diagnosis \& pathohistological diagnosis.

\begin{tabular}{|c|c|c|c|c|c|c|}
\hline & \multirow{2}{*}{ Diagnosis } & \multicolumn{4}{|c|}{ Microvascular proliferation } & \multirow{2}{*}{ Row tota } \\
\hline & & None & Low & Medium & High & \\
\hline Count & Chronic apical periodontitis & 6 & 0 & 3 & 0 & 9 \\
\hline Total percent & & $16.67 \%$ & $0.00 \%$ & $8.33 \%$ & $0.00 \%$ & $25.00 \%$ \\
\hline Count & Chronic apical periodontitis with a granuloma & 1 & 2 & 11 & 2 & 16 \\
\hline Total percent & & $2.78 \%$ & $5.56 \%$ & $30.56 \%$ & $5.56 \%$ & $44.44 \%$ \\
\hline Count & Chronic apical periodontitis with a fistula & 5 & 0 & 1 & 0 & 6 \\
\hline Total percent & & $13.89 \%$ & $0.00 \%$ & $2.78 \%$ & $0.00 \%$ & $16.67 \%$ \\
\hline Count & Chronic apical periodontitis with parulis & 3 & 1 & 1 & 0 & 5 \\
\hline Total percent & & $8.33 \%$ & $2.78 \%$ & $2.78 \%$ & $0.00 \%$ & $13.89 \%$ \\
\hline Count & All groups & 15 & 3 & 16 & 2 & 36 \\
\hline Total percent & & $41.67 \%$ & $8.33 \%$ & $44.44 \%$ & $5.56 \%$ & \\
\hline
\end{tabular}

Table 2. Chronic apical periodontitis \& microvascular proliferation.

\begin{tabular}{ccccc}
\hline \multirow{2}{*}{ Chronic apical periodontitis } & \multicolumn{2}{c}{ Microvascular proliferation } & Total \\
\cline { 2 - 4 } & Yes & Yes & No & 9 \\
& & 3 & $16.667 \%$ & $25.000 \%$ \\
No & $8.333 \%$ & 9 & 27 & $75.000 \%$ \\
Total & 18 & $25.000 \%$ & 36 \\
& $50.000 \%$ & 15 & $\mathbf{4 1 . 6 6 7 \%}$ & \\
\hline
\end{tabular}

total of 27 (75.00\%) children had some other diagnosis, from which 18 (50.00\%) children had microvascular proliferation and $9(25.00 \%)$ children did not have microvascular proliferation. The results showed that $\mathrm{p}>0.05$

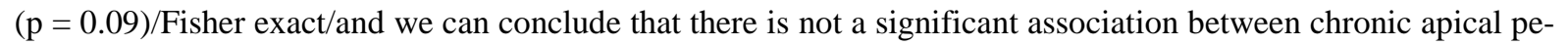
riodontitis and microvascular proliferation. The children that had chronic apical periodontitis had a 0.25 times $(\mathrm{OR}=0.25 / \mathrm{Wald} 95 \% \mathrm{Cl}: 0.05-1.24)$ smaller risk of having microvascular proliferation in comparison to the children who had other diagnosis.

The data in Table 3 show the association between the chronic apical periodontitis with a granuloma and microvascular proliferation. From a total of 36 children who had chronic apical periodontitis with a granuloma, 15 (41.67\%) children had microvascular proliferation and 1 (2.78\%) child did not have microvascular proliferation. A total of 20 (55.56\%) children had some other diagnosis, from which 6 (16.67\%) children had microvascular proliferation and $14(38.89 \%)$ children did not have microvascular proliferation. The results showed that $\mathrm{p}<$ $0.001(\mathrm{p}=0.0001) /$ Fisher exact/and that there is a significant association between chronic apical periodontitis with a granuloma and microvascular proliferation. The children who had chronic apical periodontitis with a granuloma had a 35 times (OR = 35/Wald 95\%Cl: 3.73 - 328.38) higher risk of having microvascular proliferation in comparison to the other children who had other diagnosis.

The data in Table 4 show the association between the chronic apical periodontitis with a fistula and microvascular proliferation. From a total of 36 children, chronic apical periodontitis with a fistula had 6 (16.67\%) children from which 1 (2.78\%) child had microvascular proliferation and $5(13.89 \%)$ children did not have microvascular proliferation. A total of 30 (83.33\%) children had some other diagnosis from which 20 (55.56\%) children had microvascular proliferation and 10 (27.78\%) children did not have microvascular proliferation. The 
results showed that $\mathrm{p}>0.05(\mathrm{p}=0.006) /$ Fisher exact/and that there is no significant association between chronic apical periodontitis with a fistula and microvascular proliferation. The children who had chronic apical periodontitis with a fistula had a 0.10 times $(\mathrm{OR}=0.10 /$ Wald $95 \% \mathrm{Cl}$ : $0.01-0.98)$ lower risk of having microvascular proliferation in comparison to the other children who had other diagnosis.

The data in Table 5 show the association between the chronic apical periodontitis with parulis and microvascular proliferation. From a total of 36 children, chronic apical periodontitis with parulis had $5(13.89 \%)$ children from which 2 (5.56\%) child had microvascular proliferation and 3 (8.33\%) children did not have microvascular proliferation. A total of 31 (86.11\%) children had some other diagnosis from which 19 (55.56\%) children had microvascular proliferation and 12 (33.33\%) children did not have microvascular proliferation. The results showed that $\mathrm{p}>0 / 05(\mathrm{p}=0.34) /$ Fisher exact/and that there is no significant association between chronic apical periodontitis with parulis and microvascular proliferation. The children who had chronic apical periodontitis with parulis had a 0.42 times $(\mathrm{OR}=0.42 /$ Wald $95 \% \mathrm{Cl}$ : 0.06 - 2.90) lower risk of having microvascular proliferation in comparison to the other children who had other diagnosis.

Table 3. Chronic apical periodontitis with granulomas \& microvascular proliferation.

\begin{tabular}{|c|c|c|c|}
\hline \multirow{2}{*}{ Chronic apical periodontitis with a granuloma } & \multicolumn{2}{|c|}{ Microvascular proliferation } & \multirow[b]{2}{*}{ Total } \\
\hline & Yes & No & \\
\hline \multirow[t]{2}{*}{ Yes } & 15 & 1 & 16 \\
\hline & $41.667 \%$ & $2.778 \%$ & $44.444 \%$ \\
\hline \multirow[t]{2}{*}{ No } & 6 & 14 & 20 \\
\hline & $16.667 \%$ & $38.889 \%$ & $55.556 \%$ \\
\hline \multirow[t]{2}{*}{ Total } & 21 & 15 & 36 \\
\hline & $58.333 \%$ & $41.667 \%$ & \\
\hline
\end{tabular}

Table 4. Chronic apical periodontitis with a fistula \& microvascular proliferation.

\begin{tabular}{|c|c|c|c|}
\hline \multirow{2}{*}{ Chronic apical periodontitis with a fistula } & \multicolumn{2}{|c|}{ Microvascular proliferation } & \multirow{2}{*}{ Total } \\
\hline & Yes & No & \\
\hline \multirow[t]{2}{*}{ Yes } & 1 & 5 & 6 \\
\hline & $2.778 \%$ & $13.889 \%$ & $16.667 \%$ \\
\hline \multirow[t]{2}{*}{ No } & 20 & 10 & 30 \\
\hline & $55.556 \%$ & $27.778 \%$ & $83.333 \%$ \\
\hline \multirow[t]{2}{*}{ Total } & 21 & 15 & 36 \\
\hline & $58.333 \%$ & $41.667 \%$ & \\
\hline
\end{tabular}

Table 5. Chronic apical periodontitis with parulis \& microvascular proliferation.

\begin{tabular}{|c|c|c|c|}
\hline \multirow{2}{*}{ Chronic apical periodontitis with parulis } & \multicolumn{2}{|c|}{ Microvascular proliferation } & \multirow[b]{2}{*}{ Total } \\
\hline & Yes & No & \\
\hline \multirow[t]{2}{*}{ Yes } & 2 & 3 & 5 \\
\hline & $5.556 \%$ & $8.333 \%$ & $13.889 \%$ \\
\hline \multirow[t]{2}{*}{ No } & 19 & 12 & 31 \\
\hline & $52.778 \%$ & $33.333 \%$ & $86.111 \%$ \\
\hline \multirow[t]{2}{*}{ Total } & 21 & 15 & 36 \\
\hline & $58.333 \%$ & $41.667 \%$ & \\
\hline
\end{tabular}




\section{Discussion}

The apical periodontitis is a inflammatory and destructive process of the periradicular tissue caused by etiological agents which have endodontic origins. The apical periodontitis is most commonly caused by an endodontic infection. The primary pulp of the tooth is infected by the microflora after which it becomes necrotic. In the pulp we can find a mixed microflora in which the anaerobic bacteria predominate [12]. This polimicrobe community which is part of the root canal system has biological and pathogenic characteristics: antigenic, mitogenic, chemotaxis, histolysis and activation of the hosts cells. The microbiological agents or their products in the root canal can spread to the surrounding periapical tissue [13]. When the host comes in contact with these agents it activates its defense mechanisms which are consisted from: a couple of types of cells, intercellular communicating molescules, antibody's and effector molecules. When the hosts defense mechanism and the microbiological agents come into contact a great part of the periapical tissue is destroyed which results with the forming of the different types of apical periodontal lesions [14]. Besides the immunological defense mechanisms of the host, the body can not destroy the microbes which are located in the necrotic root canal which is outside the range of action of the immunological mechanism of the host. Knowing all of this we can conclude that the apical periodontitis is not a self limiting disease [15].

The apical periodontitis is caused consequently by an endodontic infection and it is manifested in the moments when the immunological system of the host is activated from the microorganisms which are located in the root canals [16]. The evolution of the apical periodontitis is seen upon as a dynamic battle between the microbiological factors and the immunological defense mechanisms of the host in the region between the infected radicular pulp and the periodontal ligament which process results in a local inflammation, resorption of the hard tissue, destruction of the other periodontal tissues and eventually forming different pathohistological categories of the apical periodontitis frequently named as periapical lesions [8]. The treatment for apical periodontitis, which in its bases is an infection of the root canal, is consisted of lowering the number of microbes in the root canal and prevention of a re-infection by filling the canals. The treatment has a high success rate, but still in some cases it can be unsuccessful [17]. The treatment is usually not successful in those cases where the treatment doesn't meet the standards for control and elimination of the infection. In the cases where the standards are met and the procedures are followed carefully the treatment can still be unsuccessful. This is because the root canals have regions which can't be cleaned and closed with the existing equipment, materials and techniques and the infection persists. In very little cases there are factors which are located in the inflamed periapical tissue. These factors do not allow the lesion to heal properly after the treatment [18].

The identification of the causative factors of the odontogenic infections of the primary and secondary teeth have been studied by many authors and in most cases they are classified as [12] [13] [19]:

1) Microbiological (bacteria and their toxins) which can penetrate through the dentin channels as a result of caries, milolysis, coneiformed erosions, trauma or preparation, from periodontium in periodontopathic teeth, from foramen apicale, very rarely they spread through the blood and lymph, during pyemia, flu, miliar tuberculosis exc.

2) Chemical (medicaments, materials used for fillings and the alcohol used to clean the dental cavity)

3) Mechanical, which can be: acute-intensive work with instruments and chronic-bad habits, bruxism, inadequate orthodontic braces, hot or cold drinks, galvanic microcurrent, improper and frequent use of vatalion)

4) Iatrogenic (rough usage of the rotating instruments, prolonged drying of the cavities, artificial opening of the pulp and exc.)

As a predominant pathology which is a result from the chronic odontogenic infections, are considered those teeth which had chronic apical paradontitis followed by parulis which is a localized subgingival abscess in the alveolar bone which is connected with a canal (fistula) with the chronic periapical process of the primary tooth. The remaining roots from the primary dentition with apical processes are also considered as a relevant factor that is causing the dental infection [20].

When we take the permanent teeth into consideration, as causes for these infections we can list the following: chronic apical paradontitis with a fistula, fractured teeth with a fistula which is caused by caries or dental trauma, apical periodontitis verified with Rö, remaining gangrenous teeth with periapical processes and a fistula and secondary caries with periapical processes which are caused as a result of a unsuccessful treatment of the dental caries [14] [15].

The periapical lesions which are a result of the necrotic dental pulp are one of the most common pathological 
situations which affect the alveolar bone. Although we can notice many cases of non-endodontic benign and malign lesions which are presented in the periapical region, still the most common are periapical granulomas, cysts or abscesses. The tissue from the periapical regions is sent for a pathohistological examination only in cases when we are not sure about the clinical diagnosis and it is not sent routinely for the already set clinical diagnosis [19]. The frequency of the clinical screening, which is done to see if there is benefit and also how big the benefit from the biopsy is, in correlation to setting the right diagnosis, isn't known [21]. Some studies [21] [22] show that between $0.7 \%$ and $5 \%$ of the periapical biopsies result with pathohistological results which go in conclusion. These studies are almost identical with the study we carried out where from a total of 36 children with chronic apical changes and granulomas, 9 (25\%) children had chronic apical paradontitis, from which 6 (16.67\%) children did not have inflammation, 1 (2.78\%) child had a medium level of inflammation and 2 (5.56\%) children had severe inflammation.

Total 16 (44.44\%) children had chronic apical peridontitis with a granuloma from which 1 (2.78\%) child did not have inflammation, 4 (11.11\%) children had a low level of inflammation, 6 (16.67\%) children had a medium level of inflammation, and 5 (13.89\%) children had severe inflammation. A total of 6 (16.67\%) children had chronic apical periodontitis with a fistula from which 5 (13.89\%) children did not have inflamation and 1 (2.78\%) child had a medium level of inflamation.

Ricucci's [23] studies are consisted of 106 biopsies taken with apicoectomy or after an extraction of the tooth from the root canals from the teeth with apical periodontitis (64 treated and 42 untreated) which were sent for pathohistological and pathobacteriological analysis and the results from the biopsies were in conclusion with the clinical diagnosis.

In order to confirm the benefit of the histopathological examination as a confirmation of the clinical diagnosis of the odontogenic infection, Osawa [23] and col. routinely took biopsies from periradicular lesions of extracted teeth in 136 patients. The results confirmed the existence of periradicular lesions in 75 (55.2\%) patients, the existence of a periapical abscess in 30 (22.8\%) patients and the existence of granulomas in 23 (16.9\%) patients. The conclusion was that there is a great correlation between the clinical and histopathological diagnosis.

We should accent that the orofacial infections are common causes for visiting a dentist throughout the world. There is very little data about the clinical-epidemiological profiles which are published by dentist from the primary health care and for this reason we accented our focus on this problem.

Taking into consideration the frequency of the odontogenic infections in children we hope that the results from our study will be a contribution to the dental community for confirming the right diagnosis of the chronic dental infections which are confirmed with pathohistological results

\section{References}

[1] Federation Dentaire Internationale (1982) Goals for Oral Health in the Year 2000. British Dental Journal, 152, 21-22. http://dx.doi.org/10.1038/sj.bdj.4804729

[2] Beloica, D. Vulovic, M., Gajic, M. Stevanovic, R., Ivanovic, M., Carevci, M., Vuliqevic, Z.R. and Markovic, D. (2003). Decja Stomatologija, Beograd.

[3] A Review of the Developmental Defects of Enamel Index (DDE Index). Commission on Oral Health, Research \& Epidemiology. Report of an FDI Working Group (1992) International Dental Journal, 42, 411-426. http://www.biomedcentral.com/1472-6831/13/21

[4] Federation Dentaire Internationale (1982) Goals for Oral Health in the Year 2000. International Dental Journal, 32, 74-77.

[5] Commission on Oral Health (2002) Research and Epidemiology. Review of Dental Health, 19, 224-229.

[6] Seow, W.K. (2003) Diagnosis and Management of Unusual Abscess in Children. Australian Dental Journal, 48, 156168.

http://www.ijri.org/article.asp?issn=0971-3026; year=2005; volume=15;issue=3; spage=399; epage=400; aulast=Verma http://dx.doi.org/10.1111/j.1834-7819.2003.tb00026.x

[7] Chow, A.W., Roser, S.M. and Brady, F.A. (1998) Orofacial Odontogenic Infections. Annals of Internal Medicine, 88, 392-402.

[8] Gray, M.M., Marchment, M.D. and Anderson, R.J. (1991) The Relationship between Caries Experience in the Deciduous Molars at 5 Years and in First Permanent Molars of the Same Child at 7 Years. Community Dental Health, 8, 3-7. http://www.biomedcentral.com/1472-6831/14/74

[9] Stashenko, P. (1990) The Role of Immune Cytokines in the Pathogenesis of Periapical Lesions. Endodontics \& Dental 
Traumatology Journal, 6, 89-96. http://dx.doi.org/10.1111/j.1600-9657.1990.tb00400.x

[10] Nair, P.N.R., Pajarola, G. and Schroeder, H.E. (1996) Types and Incidence of Human Periapical Lesions Obtained with Extracted Teeth. Oral Surgery, Oral Medicine, Oral Pathology, 81, 93-102. http://dx.doi.org/10.1016/S1079-2104(96)80156-9

[11] Schulz, M., von Arx, T., Altermatt, H.J. and Bosshardt, D. (2009) Histology of Periapical Lesions Obtained during Apical Surgery. Journal of Endodontics, 35, 634-642. http://dx.doi.org/10.1016/j.joen.2009.01.024

[12] Leonardo, M.R., Rossi, M.A., Silva, L.A., Ito, I.Y. and Bonifacio, K.C. (2002) EM Evaluation of Bacterial Biofilm and Microorganisms on the Apical External Root Surface of Human Teeth. Journal of Endodontics, 28, 815-818. http://dx.doi.org/10.1097/00004770-200212000-00006

[13] Sunde, P.T., Olsen, I., Lind, P.O. and Tronstad, L. (2000) Extraradicular Infection: A Methodological Study. Endodontics \& Dental Traumatology Journal, 16, 84-90. http://dx.doi.org/10.1034/j.1600-9657.2000.016002084.X

[14] Sunde, P.T., Tronstad, L., Eribe, E.R., Lind, P.O. and Olsen, I. (2000) Assessment of Periradicular Microbiota by DNA-DNA Hybridization. Endodontics \& Dental Traumatology Journal, 16, 191-196. http://dx.doi.org/10.1034/j.1600-9657.2000.016005191.x

[15] Gatti, J.J., Dobeck, J.M., Smith, C., White, R.R., Socransky, S.S. and Skobe, Z. (2000) Bacteria of Asymptomatic Periradicular Endodontic Lesions Identified by DNA-DNA Hybridization. Endodontics \& Dental Traumatology Journal, 16, 197-204. http://dx.doi.org/10.1034/j.1600-9657.2000.016005197.x

[16] Graunaite, I., Lodiene, G. and Maciulskiene, V. (2012) Pathogenesis of Apical Periodontitis: A Literature Review. Journal of Oral \& Maxillofacial Research, 2, 1011-1023.

[17] Caste, L.M., Selwitz, R.H., Oldakowski, R.J., Brunelle, J.A., Winn, D.M. and Brrown, L.J. (1996) Coronal Caries in the Primary and Permanent Dentition of Children and Adolescents 1-17 Years of Age: United States 1988-1991. Journal of Dental Research, 75, 631-641.

[18] Nair, P.N.R. (1997) Apical Periodontitis: A Dynamic Encounter between Root Canal Infection and Host Response. Journal of Periodontology, 13, 121-148. http://dx.doi.org/10.1111/j.1600-0757.1997.tb00098.x

[19] da Silva L.A.B., Leonardo, M.R., de Oliveira, D.S.B. and da Silva, R.A.B. (2010) Alexandra Mussolino de Queiroz, Patrícia Gatón Hernández, Paulo Nelson-Filho. Histopathological Evaluation of Root Canal Filling Materials for Primary Teeth. Brazilian Dental Journal, 21, 38-45.

[20] Hunt, D.E., King, T.J. and Fuller, G.E. (1978) Antibiotic Susceptibility of Bacteria Isolated from Oral Infections. Journal of Oral and Maxillofacial Surgery, 36, 527.

[21] Rani, A. and Chopra, A. (2006) Isolation and Identification of Root Canal Bacteria from Symptomatic Nonvital Teeth with Periapical Pathosis. Endodontology, 18, 1-48

[22] Osawa, F.O., Bircich, D. and Onatoly, A. (2009) Clinicopathologic Correlation in the Diagnosis of Periradicular Lesions of Extracted Teeth. Journal of Oral and Maxillofacial Surgery, 64, 1387-1391.

[23] Ricucci, D. and Siqueira Jr., J.F. (2010) Biofilms and Apical Periodontitis: Study of Prevalence and Association with Clinical and Histopathologic Findings. Journal of Endodontics, 36, 345-352.

http://dx.doi.org/10.1016/j.joen.2010.04.007 